\title{
Named entity recognition in medical texts in Russian using deep learning models
}

\author{
I.V. Moskalev \\ ASU, Barnaul, Russia \\ moskalev.igor.v@gmail.com
}

\begin{abstract}
The application of contextual and domain-specific pre-trained word embeddings for recognition of medical concepts in free-text clinical notes in Russian is considered. As it is known, a large amount of medical data is stored in electronic form, a significant part - in an unstructured form (medical history, extracts, description of the results of various tests). This data contains a large amount of useful information for the diagnosis of diseases. The results of the experiments showed the effectiveness of applying contextual language models which pre-trained on medical texts in the task biomedical named entity recognition.
\end{abstract}

Keywords - information extraction of medical data; medical text processing; biomedical named entity recognition; deep learning; word embeddings

\section{Motivation and Aim}

\section{Motivation}

In recent years, relevant scientific areas in medicine are: the transition to advanced digital, intelligent technologies, the creation of large-scale data processing systems, the development of machine learning and artificial intelligence, the transition to personalized medicine, high-tech healthcare and health-saving technologies. One of the current developing areas of artificial intelligence application is the analysis of medical data and the creation of clinical decision support systems.

There is a large amount of medical data in electronic form [1]. A significant part of this data is stored in an unstructured form (health records, discharge summaries, results of various tests and procedures). However, this data contains a large amount of information.

Medical texts are characterized with non-academic text style, the presence of a large number of acronyms and abbreviations, and incomplete sentences. One of the important tasks in the analysis of medical texts is the biomedical named entity recognition (BioNER). A large number of studies are devoted to this problem in the English language [2]-[4]. But also there are a number of works for texts in Russian [5]. This paper presents the application of advanced approaches of text processing in the task of BioNER in Russian texts.

Aim

Medical data contains important information about patients. Results of tests are stored mainly in a structural form. Data such as health histories, medical examinations reports, results of procedures have an unstructured form (natural language text). The article shows the advantage of applying contextual and domain-specific pre-trained deep learning language models in biomedical named entity recognition.

\author{
L.A. Khvorova \\ ASU, Barnaul, Russia \\ khvorovala@gmail.com
}

Methods

A number of approaches were considered including the applying of contextual versus non-contextual word embeddings, and pre-training on general domain texts versus domain-specific texts. We compared the performance of noncontextual word embeddings such as word2vec, GloVe, FastText and contextual word embeddings such as ELMo and BERT. Also we experimented with general domain and domain-specific pre-training. For general domain pre-training we used data from the Russuan part of Wikipedia Corpus and Corpus of Russian news articles collected from Lenta.Ru. For domain-specific pre-training we used pages of Russian part of Wikipedia Corpus which titled medical concepts and texts of the clinical protocols of the Ministry of Health. To perform medical concepts identification we used the Bi-LSTM+CRF model with non-contextual and ELMo embeddings. In case of using BERT-based models we added a simple linear layer or Bi-LSTM layer for classification. For the final optimization and evaluation of the models, an annotated dataset was prepared. It contains about 1000 free-text clinical notes manually annotated with Brat annotation tool. For the evaluation we calculated precision, recall and F1 score.

\section{Results}

The paper assesses the quality of developed methods and models of extracting information from clinical texts in Russian. The use of data mining and processing techniques will allow to automate the solution of many medical problems arising in clinical practice, thus improving the quality of primary medical care. The results of the research show the effectiveness of using contextual and domain-specific pre-trained language models in the biomedical named entity recognition task which indicates the prospect of using developed models for clinical decision support systems.

\section{REFERENCES}

[1] P. B. Jensen, L. J. Jensen, and S. Brunak, "Mining electronic health records: Towards better research applications and clinical care," Nat. Rev. Genet., vol. 13, no. 6, pp. 395-405, Jun. 2012.

[2] B. De Bruijn, C. Cherry, S. Kiritchenko, J. Martin, and X. Zhu, "Machine-learned solutions for three stages of clinical information extraction: the state of the art at i2b2 2010," Journal of the American Medical Informatics Association, vol. 18, no. 5, pp. 557-562, 2011.

[3] S. Jonnalagadda, T. Cohen, S. Wu, and G. Gonzalez, "Enhancing clinical concept extraction with distributional semantics," Journal of Biomedical Informatics, vol. 45, no. 1, pp. 129-140, 2012.

[4] M. Habibi, L. Weber, M. Neves, D. L. Wiegandt, and U. Leser, "Deep learning with word embeddings improves biomedical named entity recognition," Bioinformatics, vol. 33, no. 14, pp. i37-i48, 2017.

[5] A. O. Shelmanov, I. V. Smirnov, E. A. Vishneva, "Information extraction from clinical texts in Russian," Computational Linguistics and Intellectual Technologies: Papers from the Annual International Conference "Dialogue", vol. 14, no. 21, pp. 537-549, 2015. 